\title{
De Novo Cavernoma in the Sensory Cortex Causing Cervicobrachialgia
}

\author{
Dhruv Jain, MS*, Michael Broduhn, MD, Sophie Rosahl and Steffen K Rosahl, MD, PhD
}

Department of Neurosurgery, Helios Klinikum Erfurt, Thuringen, Germany

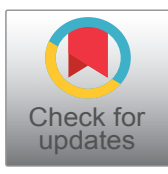

*Corresponding author: Dr. Dhruv Jain, MS, Department of Neurosurgery, Helios Klinikum Erfurt, Erfurt, Thuringen, Germany, Tel: +919-811-223-568

\begin{abstract}
Background: We report a case of rapidly expanding de novo cavernoma in the sensory cortex caus-ing/producing/ evoking cervicobrachialgia.

Medical course: A 32-year-old female presented with neck pain radiating to the ulnar forearm along with occasion-al tingling sensations. Three years earlier, she had suffered from left-sided cerebellar infarction caused by dissection of the left vertebral artery. The dissection was provoked by manual therapy administered by a certified physiotherapist. Antiplatelet treatment with Aspirin $100 \mathrm{mg} /$ day was established. One year later, she experienced tingling in the left side of her face. At the time, intracerebral micro-hemorrhage in the right sensory cortex was seen on cranial computed tomography $(\mathrm{CCT})$ and Aspirin was discontinued. A routine follow-up magnetic resonance imaging (MRI) two years later showed an increase of the hemorrhagic lesion, suggestive of a de novo cavernous hemangioma. In a follow-up MRI three months later the lesion had almost tripled in size and the patient was advised to consult a neurosurgeon. Days before the neurosurgical consultation, previously mild pain that irradiated from the right side of her neck to the ulnar side of the right arm increased sharply. Following microsurgical excision of the cavernoma, the symptoms decreased gradually. An MRI of the cervical spine was normal with no signs of disc herniation or nerve compression.
\end{abstract}

Conclusion: On rare occasions, lesions in the respective area of the somatosensory cortex may cause cervicobra-chialgia.

\section{Keywords}

Cavernous angioma, Cervicobrachialgia, Sensory cortex

\section{Introduction}

Cerebral cavernous angiomas (synonym: cerebral cavernous malformations; cavernomas) are the third most common cerebral vascular malformation after developmental venous anomaly and capillary telangiectasia [1]. Cavernomas are comprised of clusters of tightly packed, abnormally thin-walled blood vessels that displace normal neurological tissue in the brain or spinal cord [2]. Three gene mutations (CCM1, CCM2, and $\mathrm{CCM} 3$ ), which can either be inherited or occur sporadically, have been associated with the formation of cavernomas [3].

Intracerebral hemorrhage is the initial complication in $30 \%$ of cases $[4,5]$. Superficial cavernomas are associated with a lower risk of hemorrhage than those located in the depth. Patients with asymptomatic cavernomas with typical imaging findings are usually managed conservatively with serial imaging studies. Symptomatic patients may present with acute headache, epilepsy and acute or subacute neurological deficits owing to overt intra or extralesional hemorrhage [6].

We report the case of a 32-year-old female who suffered from symptoms classified as cervicobrachialgia that were caused by a rapidly growing de novo cavernoma located in the post central gyrus.

\section{Case Report}

\section{Medical history}

A 32-year-old left-handed female presented with neck pain in the area of the lateral triangle on the right, radiating to the ulnar forearm. The pain went along with occasional tingling sensations and was increased by raising the right arm. The symptoms were independent of head movements. There were no complaints of headache, nausea or vomiting.

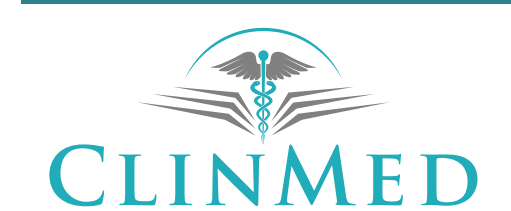

INTERNATIONAL LIBRARY ir
Citation: Jain D, Broduhn M, Rosahl S, Rosahl SK (2020) De Novo Cavernoma in the Sensory Cortex Causing Cervicobrachialgia. Neurosurg Cases Rev 3:050. doi.org/10.23937/2643-4474/1710050

Accepted: December 15, 2020; Published: December 17, 2020

Copyright: (C) 2020 Jain D, et al. This is an open-access article distributed under the terms of the Creative Commons Attribution License, which permits unrestricted use, distribution, and reproduction in any medium, provided the original author and source are credited. 

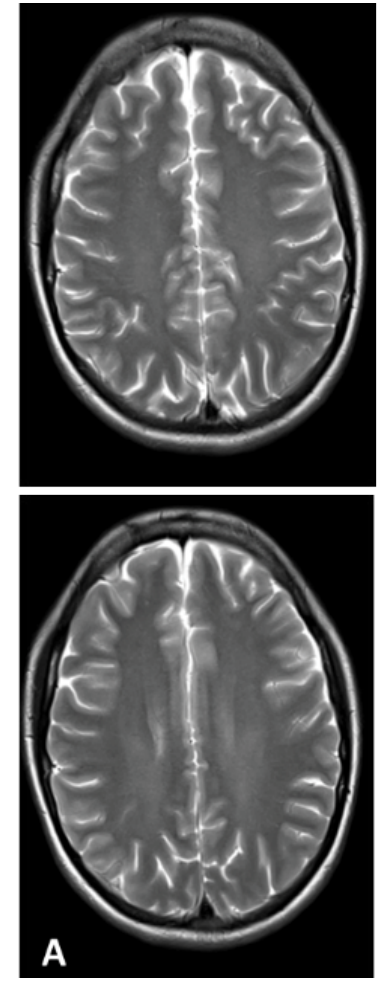
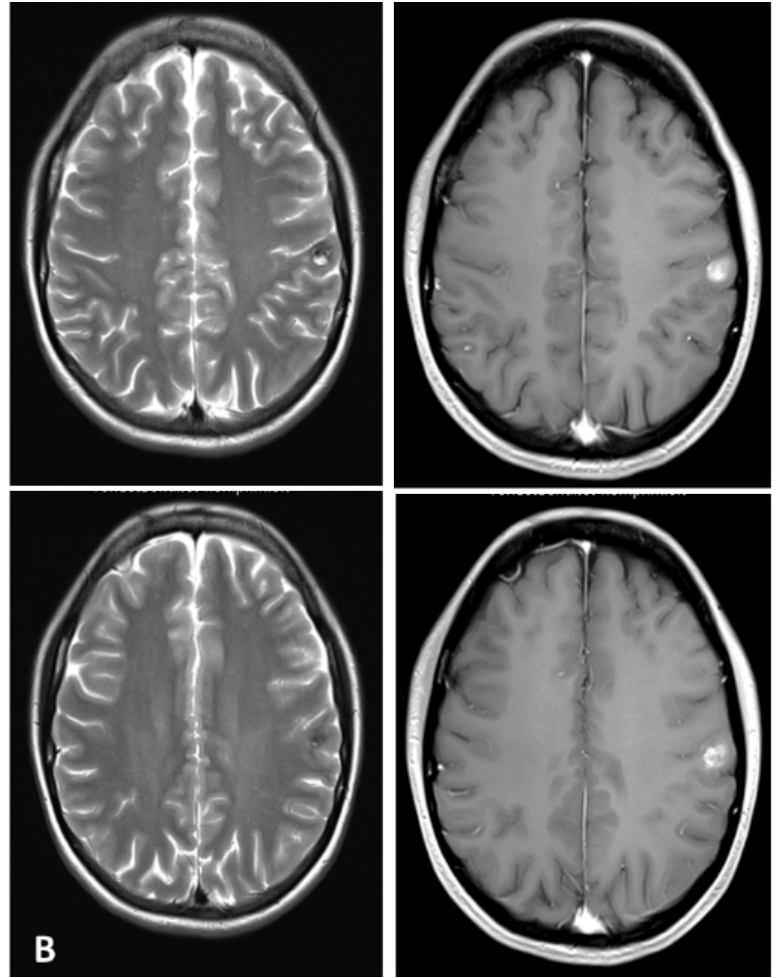
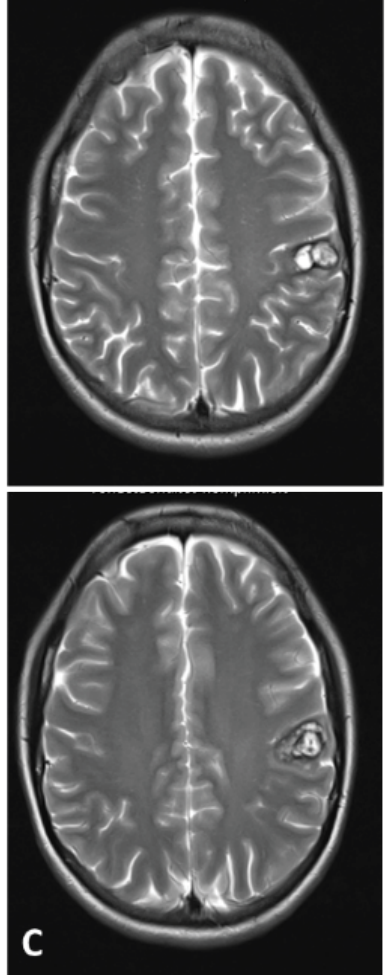

Figure 1: (A) T2-weighted MRI taken in Dec 2017, showing no lesion in the somatosensory cortex; (B) T2- and post-contrast T1-weighted images taken in Jan 2017. De novo cavernoma in the left somatosensory cortex; (C) Follow-up T2-weighted MRI three months later, the lesion had tripled in volume and the patient suffered from left-sided cervicobrachialgia and dysesthesia.

In September 2017, the patient had suffered from left-sided cerebellar infarction caused by dissection of the left vertebral artery following manual therapy administered by a certified physiotherapist. Thereafter, antiplatelet treatment with $100 \mathrm{mg} /$ day of Aspirin was started. The patient was oligosymptomatic until November 2018, when she reported an isolated tingling sensation on the left side of her face. At that time, intracerebral micro-hemorrhage was seen on the CCT and Aspirin was discontinued.

In January 2020, the patient reported minor holocranial headache (VAS 3/10) for the first time. An MRI showed a hyperdense lesion in the left postcentral gyrus which was classified as a small cavernous angioma by a radiologist. In March, the patient reported painful dysesthesia in the right lateral cervical triangle, radiating over the lateral upper arm to the ulnar forearm. The tendon of the trapezius muscle was palpably and even visibly tense on the right side. Another follow-up MRI was performed on April 20, which showed an increase in size of the lesion to about three times the previous size.

\section{Neurological examination}

On examination, the left-handed patient showed a prominent and tense superior tendon of the trapezius muscle on the right side. Algophobic weakness of the triceps muscle on the right side. During examination the pain was worsened by both actively and passively raising the right arm. All symptoms were independent of head movements. No other motor or sensory deficit was identified.

\section{Radiological findings}

In December 2017, apart from showing the circumscript postischemic lesion in the left cerebral hemisphere, an MRI of the head did not reveal any significant abnormalities (Figure 1A). On January 22, 2020, a small, contrast-enhancing hemorrhagic lesion could be seen in the left postcentral gyrus, more precisely in the somatosensoric area representing the right arm (Figure $1 \mathrm{~B}$ and Figure 2). In April 2020, the lesion had tripled in volume (Figure $1 \mathrm{C}$ ).

The MRI of the cervical spine did not show any signs of compression of neural structures (Figure 3).

\section{Intervention}

With the patient in supine position, a left parietal craniotomy was performed under image-guidance. A microsurgical approach with a corticotomy at the site where the lesion was closest to the surface was chosen, and intraoperative ultrasound was employed as a second guidance system. For additional safety, reversal somatosensensory potentials were measured before the corticotomy was carried out. The cavernoma was anatomically dissected from the surrounding somatosensory cortex and removed entirely without complications.

\section{Histological examination}

Histological sections of the cavernous hemangioma showed numerous back-to-back, partly capillary, part- 


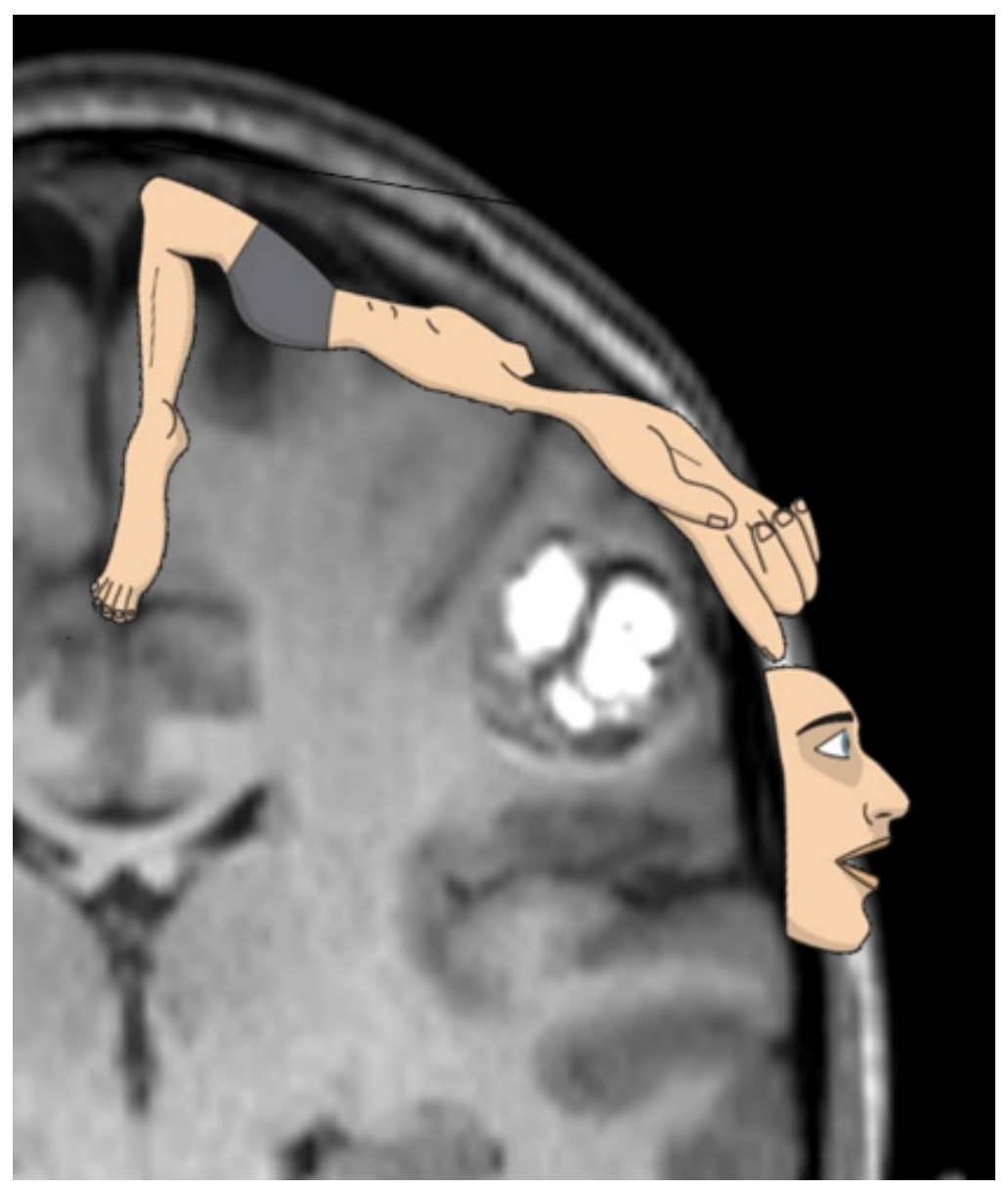

Figure 2: Coronal section of the T1-weighted, contrast-enhanced MRI showing the cavernoma before surgery. On overlay of the schematic homunculus on the somatosensory cortex shows the lesion to be in the area corresponding to the sensory representation of the arm and the hand.

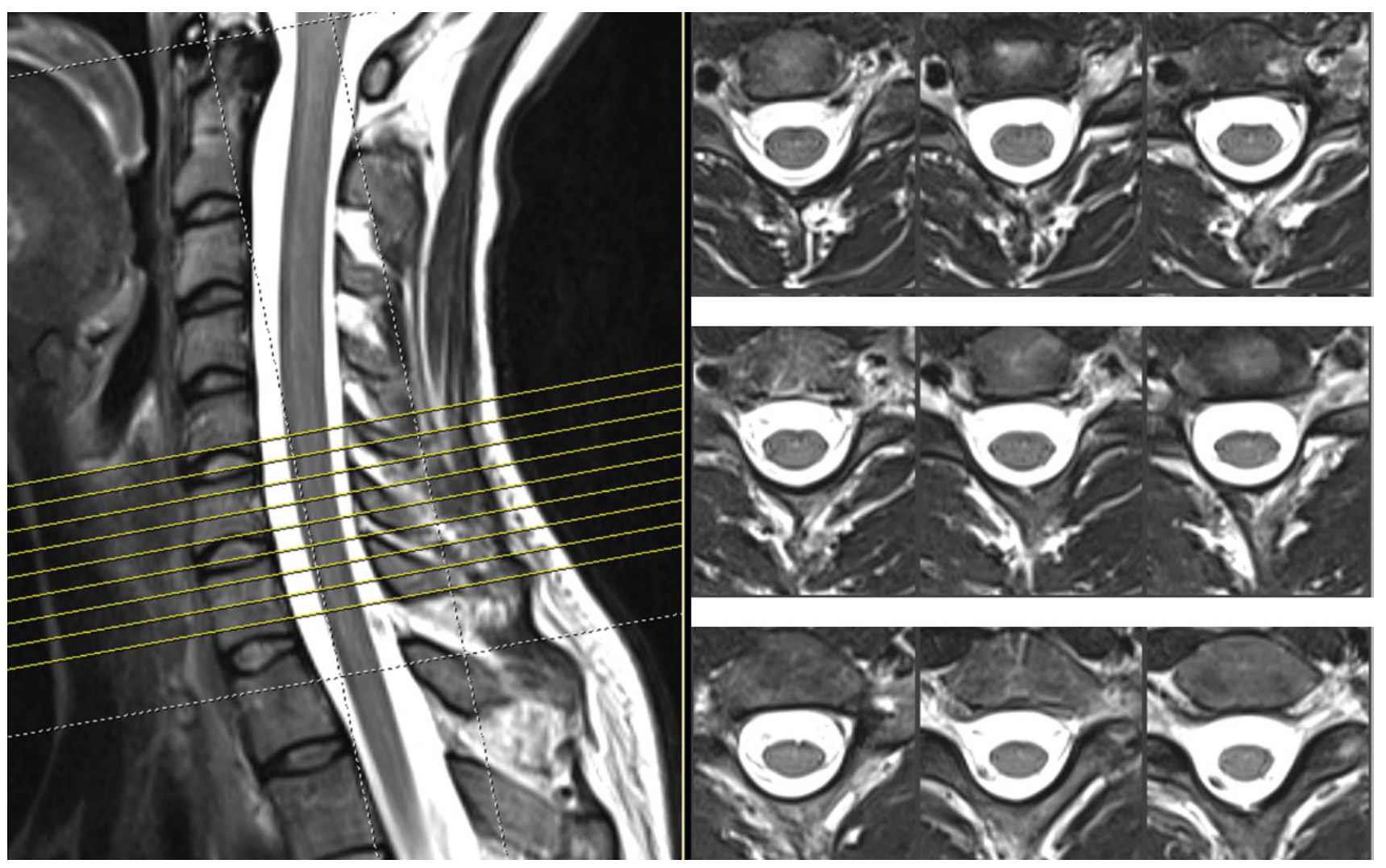

Figure 3: Cervical spine MRI of the patient showing normal radiological findings. 


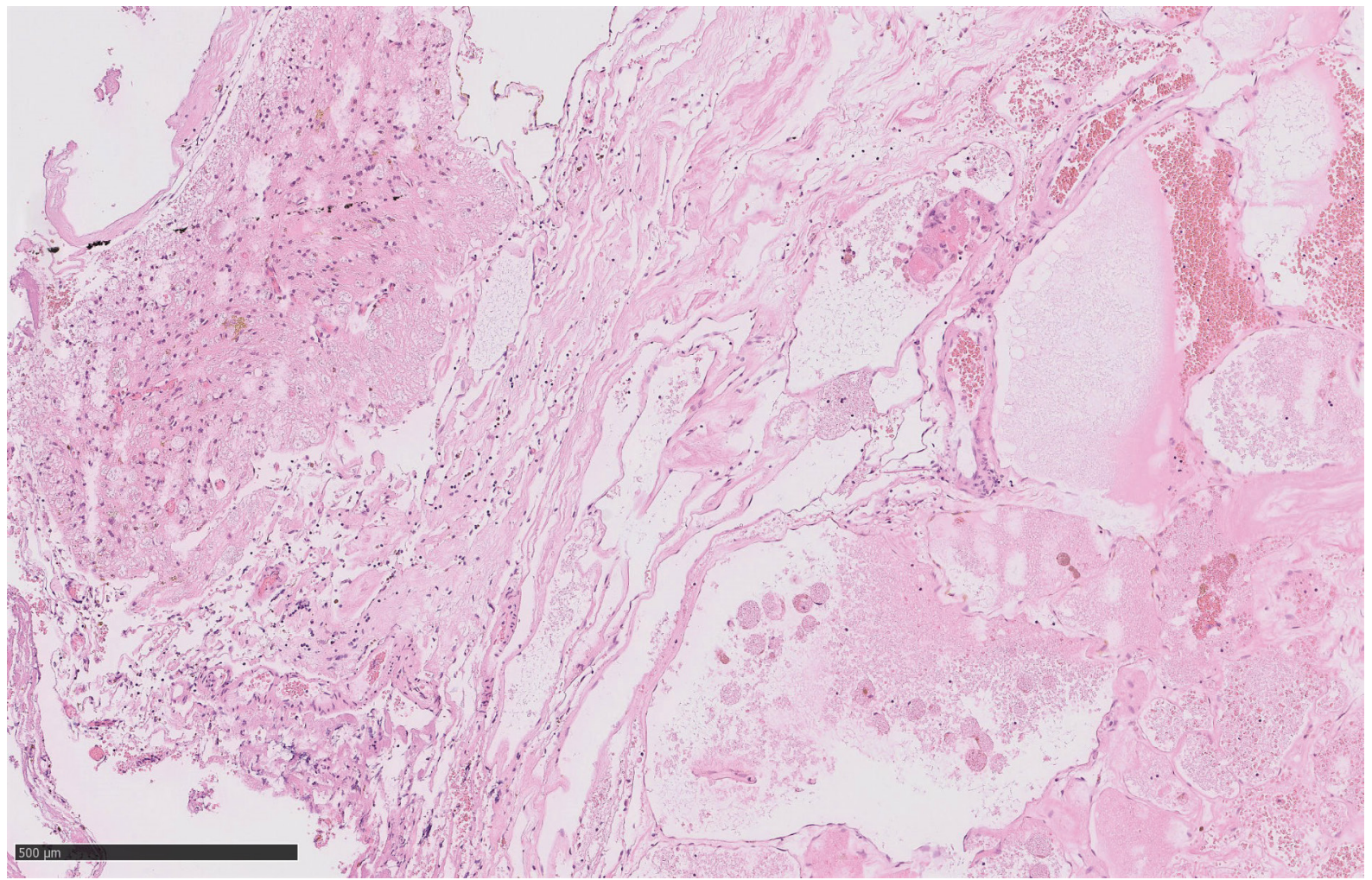

Figure 4: Hematoxylin-Eosin stained histological section through the surgical specimen of the lesion showing the typical features of a cavernoma with multiple, partly capillary, partly cavernous dilated blood vessels with abundant surrounding hemosiderophage accumulations and moderate lymphohistiocytic inflammation (WHO grade I).

ly cavernous, dilated blood vessels with abundant surrounding hemosiderophage accumulations and moderate lymphohistiocytic inflammation caused by relapsed hemorrhages and surrounding reactive piloid astrogliosis. No atypia was seen in endothelial cells or the pericytes (Figure 4).

\section{Postoperative course}

Postoperatively, the cervicobrachialgia resolved gradually and seized completely one month later. An MRI at three months post-surgery showed hemosiderin residuals in the area of previous surgery. Contrast-enhanced scans did not reveal any remnant of the cavernoma.

\section{Discussion}

Cavernomas are more common type of intracerebral vascular lesions than is generally suspected. They are comprised of clusters of tightly packed, abnormally thin-walled, small blood vessels that displace normal neurological tissue in the brain or spinal cord $[1,7]$. In recent literature, the prevalence of cavernomas has been reported to be between $0.1 \%$ and $0.8 \%[3,8]$.

In most cases, cavernomas occur sporadically with isolated lesions. The familial form is characterised by multiple lesions with an autosomal dominant mode of inheritance. Cavernomas have also been long known to calcify and to develop 'de novo' from microscopic arteriovenous lesions [9].
Histologically, the defective blood vessels in cavernomas lack pericytes and tight and adherent junctions resulting in a single layer lining consisting of endothelial cells. This leads to the impairment of the blood-brain barrier [10].

In the brain and spinal cord, cavernomas can be fragile and lead to bleeding, causing hemorrhagic stroke, seizures, and neurological deficits. They can range in size from a few fractions of an inch to several inches in diameter, depending on the number of blood vessels involved. Potential risk factors for hemorrhage include sex (females > males), age, lesion location, size, trauma, perfusion, and prior hemorrhage $[11,12]$.

Common clinical manifestations are intralesional or extralesional intracerebral hemorrhage with or without focal neurological deficits and seizures $[8,13]$.

In the case presented here, clinical symptoms included radicular pain and dysesthesia in the upper limb, closely resembling cervicobrachialgia caused by degenerative lesions of the cervical spine. This was particularly suggestive because the patient had a history of neck pain that had been treated by manual therapy years before. Tragically, the physiotherapeutical intervention at that time led to dissection of the vertebral artery and consecutive embolic cerebellar infarction.

Cervicobrachialgia is a term that describes pain and stiffness of the cervical spine with symptoms in the shoulder girdle and upper extremity [14]. It can be associated with tingling, numbness or discomfort in the arm, 
upper back and upper chest with or without an associated headache.

Our patient presented with all these symptoms. If it was not for her medical history that included the cerebellar ischemia which had been monitored through a follow-up MRI, an intracerebral cause of her symptoms would probably not have been suspected primarily. Diagnostic examination would probably have stopped at cervical imaging. Physical therapy might have been initiated and - in light of the patient's vulnerability with respect to vertebral artery dissection may have led to yet another complication.

After having encountered such an unusual clinical constellation, our own awareness to the differential diagnoses of cervicobrachialgia has heightened. If the pain cannot be provoked by cervical movements, and if there is no clear radicular distribution of sensory or motor symptoms, an intracerebral lesion should be suspected. These lesions can be located in or around the somatosensory cortex [15] and may include various pathologies.

If a cavernoma is suspected, magnetic resonance imaging of the brain is the best radiological examination to detect these lesions. The cranial MRI of our patient showed that the 'de novo' cavernoma was located in the postcentral gyrus, in the somatosensory area representing the right arm and hand (Figure 2). While averaged data of how incoming sensory information from the human body is represented in the postcentral cerebral cortex have long been aggregated into a 'sensory homunculus' (Figure 2), individual sensory representation may vary. Since the tension of our patient's trapezius muscle was relieved after removal of the cavernoma, we suggest that the sensory area of the neck was also involved by the lesion.

\section{Conclusion}

On rare occasions, cervicobrachialgia can be caused by lesions in the postcentral cortex where sensory information from the contralateral upper limb and side of the neck is processed.

Even in a patient with a previously inconspicuous cerebral MRI, a 'de novo' cavernoma can rapidly increase in volume and lead to cervicobrachialgia, a symptom complex normally only found in cervical spine lesions. In patients presenting with cervicobrachialgia where no cervical abnormalities can be detected, cerebral cavernoma should be considered as a differential diagnosis and consequently excluded through a cerebral MRI.

\section{References}

1. Robinson JR, Awad IA, Little JR (1991) Natural history of the cavernous angioma. J Neurosurg 75: 709-714.

2. Fritschi JA, Reulen HJ, Spetzler RF, Zabramski JM (1994) Cavernous malformations of the brain stem. A review of 139 cases. Acta Neurochir 130: 35-46.

3. Mouchtouris N, Chalouhi N, Chitale A, Robert M, Stavropoula IT, et al. (2015) Management of cerebral cavernous malformations: From diagnosis to treatment. Scientific World Journal.

4. Zabramski JM, Wascher TM, Spetzler RF, Johonson B, Golfi- nos J, et al. (1994) The natural history of familial cavern- ous malformations: Results of an ongoing study. J Neurrosurg 80: 422-432.

5. Rigamonti D, Drayer BP, Johnson PC, Hadley MN, Zabramski J, et al. (1987) The MRI appearance of cavernous malformations (angiomas). J Neurosurg 67: 518-524.

6. Al-Shahi Salman R, Berg MJ, Morrison L, Awad IA (2008) Hemorrhage from cavernous malformations of the brain: definition and report- ing standards. Stroke 39: 3222-3300.

7. Dalyai RT, Ghobrial G, Awad I, Tjoumakaris S, Gonzalez LF, et al. (2011) Management of incidental cavernous malformations: A review. Neurosurg Focus.

8. Haasdijk RA, Cheng C, Maat-Kievit AJ, Duckers HJ (2012) Cerebral cavernous malformations: From molecular pathogenesis to genetic counselling and clinical management. Eur J Hum Genet 20: 134-140.

9. Rosahl SK, Caroline Cheng, Anneke J Maat-Kievit, Henricus J Duckers (1998) Ossified and de novo cavernous malformations in the same patient. Clin Neurol Neurosurg 100: $138-143$.

10. Spiegler S, Rath M, Paperlein C, Felbor U (2018) Cerebral cavernous malformations: An update on prevalence, molecular genetic analyses, and genetic counselling. Mol Syndromol 9: 60-69.

11. Rigamonti D, Hadley MN, Drayer BP, Johnson PC, Hoenig-Rigamonti K, et al. (1988) Cerebral cavernous malformations. Incidence and familial occurrence. N Engl J Med 319: 343-347.

12. Gross BA, Lin N, Du R, Day AL (2011) The natural history of intracranial cavernous malformations. Neurosurg Focus.

13. Chang CW, Hsu PW, Wei KC, Chang CW, Fung HC, et al. (2019) CCM1 and CCM2 variants in patients with cerebral cavernous malformation in an ethnically Chinese population in Taiwan. Sci Rep 9: 12387.

14. DeStefano LA (2011) Greenman's Principles of manual medicine. ( $4^{\text {th }}$ edn), Lippincott Williams \& Wilkins, Baltimore.

15. Khalatbari M, Ghalenoui H, Yahyavi ST, Borghei-Razavi H (2008) Left somatosensory cortex tumor presented with radicular hand pain and paresthesia. Arch Iran Med 11: 107109. 